\title{
What's it like to live with idiopathic pulmonary fibrosis? Ask the experts
}

\author{
Anne-Marie Russell ${ }^{1}$ and Jeffrey J. Swigris ${ }^{2}$ \\ Affiliations: ${ }^{1}$ Imperial College, National Heart and Lung Institute and Royal Brompton Hospital, London, UK. \\ ${ }^{2}$ Interstitial Lung Disease Program, National Jewish Health, Denver, CO, USA.
}

Correspondence: Anne-Marie Russell, Imperial College Faculty of Medicine, National Heart and Lung Institute, Emmanuel Kaye Building, Manresa Road, London, SW3 6LR, UK. E-mail: a.russellaimperial.ac.uk

-

@ERSpublications

Sharing and informing IPF healthcare decisions through partnerships with patients and their caregivers http://ow.ly/YHp3X

Over the last 5-7 years, an enlightened paradigm has emerged for clinical research. In it, patients are not simply "subjects" to be studied or numbers to be analysed; more than "key stakeholders", patients are increasingly (and justly) recognised as the soul and substance of clinical investigation [1]. Patient advocacy organisations have added significant momentum to this movement by promoting patient-centred healthcare and helping to give a global voice to those who suffer from a range of conditions or impairments. Recent discussion on stratified medicine advocates the inclusion of patient perspectives and experience to inform the often complex decision-making processes regarding treatment [2]. Governing bodies and funding organisations have developed policies, programmes and agendas that have largely shaped the paradigm by fostering patient-centred investigations and advocating that studies be designed to ask and answer questions deemed important to patients, and include outcomes that are meaningful to patients [3].

How should a clinical investigator formulate questions that are important to patients and select outcomes that are meaningful to patients? The answer is simple: talk to the patients, the true experts of living with their condition. Informal caregivers (spouses, partners, loved ones of patients), sometimes referred to as invisible second patients because they are indirectly affected by the disease, are also experts who can provide rich insight into the patient's condition and adaptive behaviours that patients, themselves, may not see.

Qualitative research methods, often operationalised by conducting interviews and/or focus groups, enable researchers to engage the experts of the target population to capture their experiences and perceptions. Sessions are recorded, transcribed verbatim and then analysed. Whilst, historically, qualitative research has been repudiated as inferior science, there is growing recognition of the value of qualitative methods, particularly for addressing questions of understanding. Qualitative and quantitative research methods have many similarities: in each, investigators start with a question, formulate a hypothesis, recruit subjects, collect data, and then analyse and interpret the results. There are also some differences: qualitative studies focus on questions of "how" and "why", so the analytic unit is text (words, phrases and sentences), rather than numeric values generated by the quantitative perspective of "how many" or "how often".

In contrast to quantitative studies, in which protocols are necessarily cemented at the outset and remain unchanged throughout the course of the study, some qualitative investigators use a more fluid, malleable approach. For example, one or two interviews or focus groups are conducted, the data are analysed, and the findings are used to inform subsequent interviews or focus groups aimed at filling conspicuous gaps or to drill deeper into certain topics. There are a number of different approaches to qualitative analysis, each employed depending on the research hypothesis; however, regardless of approach, the over-arching objective of qualitative analysis is to answer the question "What is going on here [in the data]?" [4]. To begin, the analysts must first read and re-read the transcripts to develop familiarity with (become

Received: Jan 142016 | Accepted after revision: Feb 172016

Conflict of interest: Disclosures can be found alongside the online version of this article at erj.ersjournals.com

Copyright CERS 2016 
immersed in) the data. A systematic approach is essential to develop themes that describe what is "going on" in the data, and this is often achieved by using coding. Data are broken into manageable chunks of varying sizes (words, phrases, sentences or paragraphs), and codes (usually meaning-packed words or phrases) are applied to label concepts they represent in the chunks of data. Similar or linked concepts are ascribed the same code.

Next, the analysts attempt to group codes into broader categories (themes); this reduces the data to smaller, more manageable units that "depict the problems, issues, concerns ... of those being studied" [4]. Ultimately, if appropriate for the goals of the study, analysts develop an even broader theoretical scheme that explains the totality of the data. In the end, pages and pages of transcripts are reduced to a page (or more) of codes; codes are fitted together to generate a smaller number of themes; and themes are examined to build a theoretical framework that explains the data and answers the research question. Computer software is frequently used to manage data and track codes and themes, as well as house notes and memos that analysts generate in the course of the analysis. As one might imagine, this process is time consuming. Whilst iterative, it is free-flowing and creative, and there is some subjectivity. Working with multiple analysts minimises this. However, analysing the same set of qualitative data could certainly lead to different results, depending on the question(s) being asked. One of the benefits of qualitative analyses is that they allow investigators to "give voice" [4] to the respondents, the real experts.

In this issue of the European Respiratory Journal, OvERGAARD et al. [5] give voice to idiopathic pulmonary fibrosis (IPF) experts: they studied 24 patient/caregiver dyads (and a 25th single patient) to better understand how IPF affected the patient, the caregiver and the patient-caregiver relationship. Among the 24 caregivers, 21 were spouses or ex-spouses, and 15 were men; thus, most of the patient subjects were presumably females. Their mean age was 71 years, most were former smokers, seven used supplemental oxygen, pulmonary physiology suggested mild impairment (per cent predicted forced vital capacity and diffusing capacity were $83 \%$ and $50 \%$, respectively), 15 were taking pirfenidone and 23 were retired or disabled from work.

The investigators developed an interview guide using published literature and refined the guide after two pilot interviews. The following are examples of interview questions. How does your illness affect your life right now? What physical issues do you experience? What psychological issues do you experience? How do you support each other? How do you handle the illness? Through careful analysis, the investigators identified and named six themes: 1) information and disclosure; 2) reactional dyssynchrony; 3 ) perpetual vigilance; 4) emotional ambivalence; 5) gradual and tacit role shift; and 6) adapted coping strategies.

OVERGAARD et al. [5] used the framework method to conduct their analysis, which incorporated the process described above but also enabled thematic analysis from the perspective of the patient, the caregiver and the patient/caregiver couple [6]. The article contains an information-packed matrix within which the six themes are defined for each perspective. As individuals, patients and caregivers craved information about IPF, particularly on how it might progress. They stressed that practitioners should be attentive to the informational needs of both patients and caregivers, and particularly for patients, employ a controlled-release approach, perhaps over several visits. As couples, participants conveyed the importance of working together (in constant contact) to monitor and cope with the disease, but they also recognised that silent presence was sometimes needed. Couples stressed the possibility that their reactions to the patient being handed the diagnosis of IPF might not occur in step; for example, the patient could have moved through denial to acceptance while the caregiver was stuck in denial. Individuals within couples found themselves trying to protect each other, attempting to live normal lives, while the threat of IPF progression and death were ever-looming. They had to prepare for (or, in some cases, had already begun the process of) shifting roles within the household: the patient relinquishing duties, while the caregiver assumed them. By using dyadic interviews (having the patient and a caregiver in the same session), OVERGAARD et al. [5] promoted and observed interactions that would have been missed if the two were interviewed separately. As the authors recognise, this approach might also have had the unwanted effect of creating bias: a person may withhold information, alter answers or say only what $\mathrm{s} /$ he thinks the other person in the couple wants to hear. Despite this, the authors give readers a glimpse into the delicate ebbs and flows that permeate the IPF patient-caregiver relationship.

Other researchers have conducted qualitative studies with IPF patients or their caregivers. SCHOENHEIT et al. [7] interviewed 45 European patients with IPF and identified unmet needs in areas of disease education resources, access to centres of excellence, and family support programmes. Through interviews with IPF patients and caregivers, GIот et al. [8] identified proactive and reactive patient mindsets that appeared to drive their coping mechanisms, and they highlighted caregivers' needs for more detailed information on IPF. Russell et al. [9] further demonstrated the need for accessible, contemporary information for patients and caregivers, particularly on managing side-effects of pharmacological therapies. 
BELKIN et al. [10] observed that informal caregivers of IPF patients felt confined to an ever-shrinking world within which they struggled to find the balance between giving care and maintaining their own identity. Other qualitative work has generated calls to improve the clinical encounter with IPF patients and their caregivers, to better recognise the value of the caregiver and to respond to their different and changing needs through the trajectories of IPF [11], and to emphasise the value of peer support for IPF patients [12]. Work is underway in a research programme aimed at identifying and addressing the palliative care needs of IPF patients and their caregivers [13], whose needs are described as being similar to the needs of cancer patients' informal caregivers.

The study by OvergaARD et al. [5] reinforces and extends many of these findings. The authors reveal the struggles IPF imposes on patients and their caregivers as individuals, and on the two as couples; the results emphasise that the care of the IPF patient must be individualised, ultimately to the patient, but also include the informal caregiver to whom many patients are inextricably linked. Despite these excellent advances, some practical matters remain inadequately addressed. For example, what is the best modality to use to educate patients and caregivers about the disease, its natural history and/or nonpharmacological therapies? It is also clear that caregivers need more support. How should that support be operationalised? How can we help caregivers cope with the effects of having a loved one stricken with IPF? How do we help them prevent their worlds from shrinking as the disease progresses? How do they avoid caregiver burnout and other health hazards of providing care, particularly in the latter stages of IPF? How can we help them adapt to their ever-changing roles, as patients are forced to accept ever-declining abilities to contribute physically to the household?

Although there is much work to be done, the IPF community is realising that healthcare decisions are best made through partnerships with patients and their caregivers. If we are to partner with them to make critical decisions on important health-related matters, we had better know what they are experiencing, how those experiences affect them and whether those effects matter. This vital knowledge will only come through talking with the experts.

\section{References}

1 Office of the Legislative Counsel. Compilation of Patient Protection and Affordable Care Act: Extracted Sections Concerning Patient-Centered Outcomes Research and the Authorization of the Patient-Centered Outcomes Research Institute (PCORI). Washington, Office of the Legislative Counsel, 2010. Available from: www.pcori.org/ sites/default/files/PCORI_Authorizing_Legislation.pdf

2 Agency for Healthcare Research and Quality. Funding Opportunity Announcement (FOA) Guidance. Rockville, Agency for Healthcare Research and Quality, 2015. Available from: www.ahrq.gov/funding/policies/foaguidance/ index.html

3 Britten N, Pope C, Halford S, et al. What if we made stratified medicine work for patients? Lancet Respir Med 2016; 4: 8-10.

4 Strauss A, Corbin J. Basics of Qualitative Research: Techniques and Procedures for Developing Grounded Theory. 2nd Edn. Thousand Oaks, Sage Publications, 1998.

5 Overgaard D, Kaldan G, Marsaa K, et al. The lived experience with idiopathic pulmonary fibrosis: a qualitative study. Eur Respir J 2016; 47: 1472-1480.

6 Gale NK, Heath G, Cameron E, et al. Using the framework method for the analysis of qualitative data in multi-disciplinary health research. BMC Med Res Methodol 2013; 13: 117.

7 Schoenheit G, Becattelli I, Cohen AH. Living with idiopathic pulmonary fibrosis: an in-depth qualitative survey of European patients. Chron Respir Dis 2011; 8: 225-231.

8 Giot C, Maronati M, Becattelli I, et al. Idiopathic pulmonary fibrosis: an EU patient perspective survey. Curr Respir Med Rev 2013; 9: 112-119.

9 Russell AM, Ripamonti E, Vancheri C. Qualitative European survey of patients with idiopathic pulmonary fibrosis: patients' perspectives of the disease and treatment. BMC Pulm Med 2016; 16: 10.

10 Belkin A, Albright K, Swigris JJ. A qualitative study of informal caregivers' perspectives on the effects of idiopathic pulmonary fibrosis. BMJ Open Respir Res 2014; 1: e000007.

11 Sampson C, Gill BH, Harrison NK, et al. The care needs of patients with idiopathic pulmonary fibrosis and their carers (CaNoPy): results of a qualitative study. BMC Pulm Med 2015; 15: 155.

12 Duck A, Spencer LG, Bailey S, et al. Perceptions, experiences and needs of patients with idiopathic pulmonary fibrosis. J Adv Nurs 2015; 71: 1055-1065.

13 Bajwah S, Koffman J, Higginson IJ, et al. "I wish I knew more ..." the end-of-life planning and information needs for end-stage fibrotic interstitial lung disease: views of patients, carers and health professionals. BMJ Support Palliat Care 2013; 3: 84-90. 